\title{
A Comparison of Hard and Soft Copies Processed With Linear Conversion and Nonlinear Conversion of Skeletal Fractures-A Preliminary Study
}

\author{
Tatsumi Kaji, Michiaki Katayama, Shigeru Kosuda, and Shoichi Kusano
}

\begin{abstract}
To compare hard copies and soft copies processed with linear and nonlinear conversion of skeletal fractures, we performed a receiver-operating characteristics study of 25 digital radiographs. There was no statistical difference between hard copies with linear and nonlinear conversion. However, it may be difficult to detect small fractures on images processed by nonlinear conversion. No significant difference was seen between CRT diagnosis and that on a hard copy processed with linear conversion and frequency enhancement.

Copyright $\odot 1995$ by W.B. Saunders Company
\end{abstract}

KEY WORDS: computed radiography, hard-copy image, soft-copy image, skeletal radiology, skeletal fracture.

C OMPUTED RADIOGRAPHY is a relatively new digital radiographic system that uses a photostimulable phosphor plate as the $\mathrm{x}$-ray detector. The diagnostic accuracy of digital images, compared with analog images in chest radiography, ${ }^{1}$ mammography, and musculoskeletal radiography, ${ }^{2}$ have been assessed in a number of studies. It was reported that there is no significant difference in diagnostic accuracy between film-screen and computed musculoskeletal radiography. ${ }^{3}$ Since June 1992, in our department we have routinely used a two-on-one format, with one image produced to simulate a conventional radiograph and the other with significant edge enhancement. We are planning to deliver a single image processed with preset parameters, because a two-on-one format is small in size and not well received by other clinicians. By April 1995, an interactive workstation system connecting the emergency and radiology areas is to be introduced at our institution. The plan calls for our interpretations to be made on a workstation system in emergency cases. To our knowledge, there are few reports that compare hard copies processed by different parameters and evaluate workstations designed for diagnosis of skeletal fractures.

\section{MATERIALS AND METHODS}

\section{Case Selection}

Twenty-five digital radiographs which were obtained between June 1992 and December 1993, of 22 traumatized patients ( 8 females, 14 males) were selected at random. The patients ranged in age from 17 to 60 years (mean, 23.4). The site of fracture included the pubis $(n=15)$, ischium $(n=15)$, ilium $(n=13)$, sacrum $(n=2)$, lumbar spine $(n=4)$, rib $(n=3)$ and femur $(n=2)$, and were confirmed on computed tomography scans. The radiographic projection included the pelvis $(n=19)$ and lumbar spine $(n=6)$.

\section{Processing Conditions}

Two kinds of hard-copy images were produced for each radiograph. One was processed with linear gradation and increased enhancement (edge enhancement). The other was processed with nonlinear gradation without edge enhancement to simulate a conventional radiograph. These conditions are routinely used in our department.

\section{Image Display Functions and Requirement}

Fuji computed radiography (HI-C 654; Fuji Photo Film Co, Ltd, Tokyo, Japan) uses a 20-inch display with a resolution of 1,568 $\times 1,152 \times 8$ bits and two 32 -bit central processing units. This workstation system has a touch-panel keyboard (Fig 1) that can immediately offer an image with appropriate parameters. The same parameters as a hard copy processed with linear conversion were used for an initial image on the display system. Among the display functions, only the magnification technique was used. The

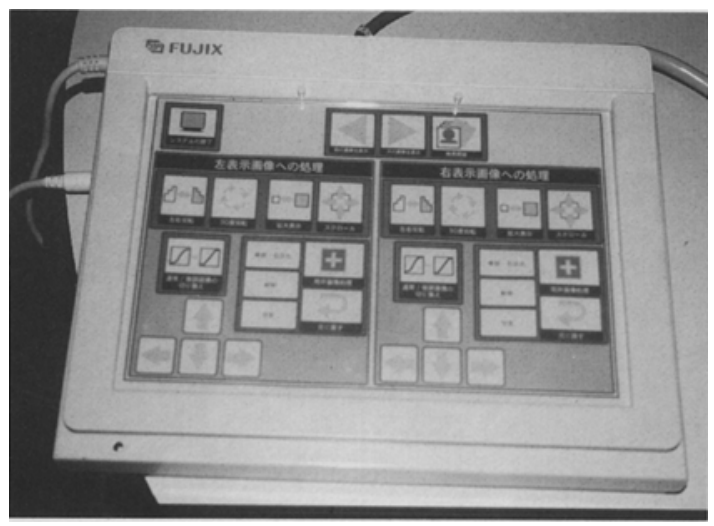

Fig 1. A touch-panel keyboard.

From the Department of Radiology, National Defense Medical College, Saitama, Japan.

Address reprint requests to Tatsumi Kaji, MD, Department of Radiology, National Defense Medical College 3-2 Namiki, Tokorozawa City, Saitama 359, Japan.

Copyright 01995 by W.B. Saunders Company

0897-1889/95/0801-1012\$3.00/0 
Table 1. Average Reading Times (min)

\begin{tabular}{cccc}
\hline & $\begin{array}{c}\text { Reader } \\
\text { No. } 1 \\
(52 \mathrm{yrs})\end{array}$ & $\begin{array}{c}\text { Reader } \\
\text { No. } 2 \\
(47 \mathrm{yrs})\end{array}$ & $\begin{array}{c}\text { Reader } \\
\text { No. } 3 \\
(30 \mathrm{yrs})\end{array}$ \\
\hline $\begin{array}{c}\text { Hard copy } \\
\text { (nonlinear) } \\
\text { Hard copy } \\
\text { (linear) } \\
\text { Workstation } \\
\text { (linear) }\end{array}$ & $1.18(.5-2)$ & $1.13(.33-1.83)$ & $1.16(.83-1.5)$ \\
\hline
\end{tabular}
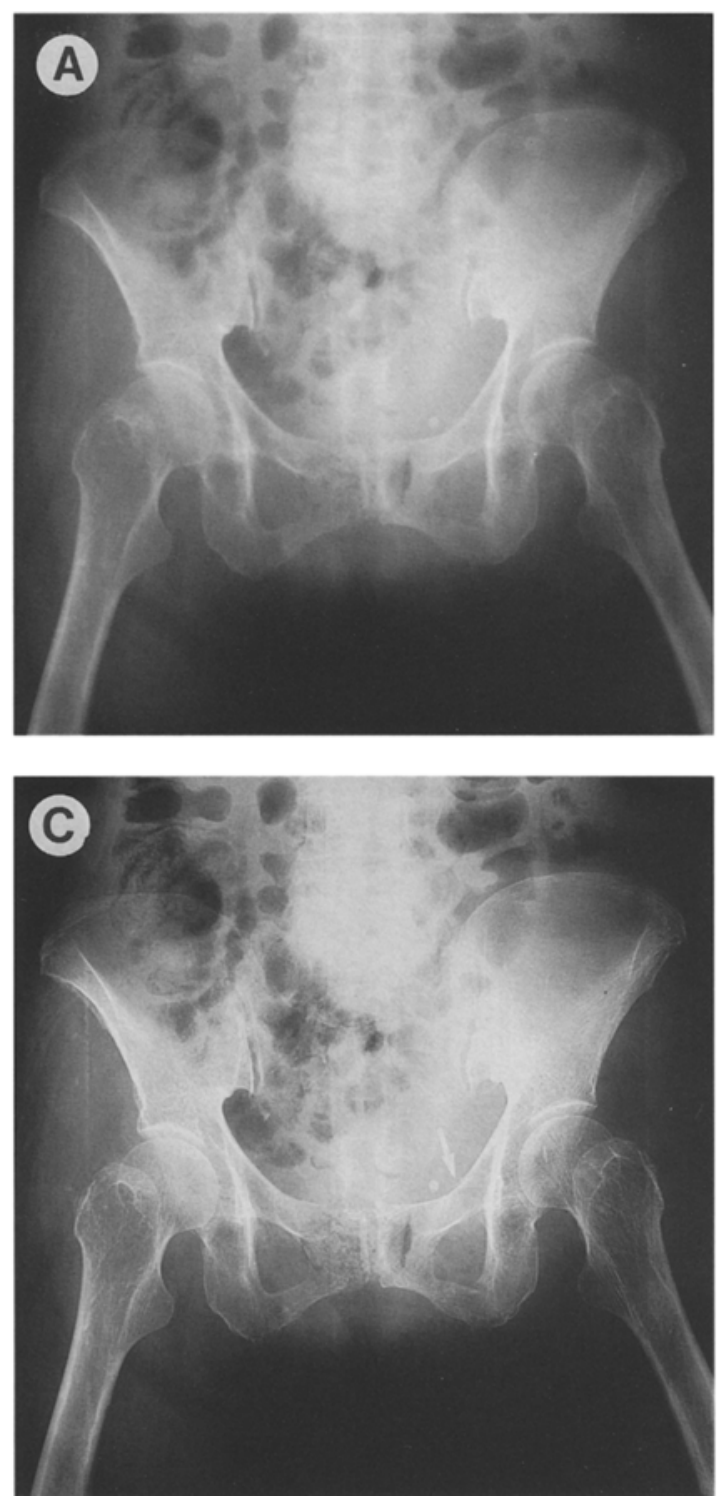

other functions such as window and level adjustment, were not used.

\section{Image Interpretation}

Three radiologists, aged 52,47 , and 30 years, participated as readers. One was experienced, whereas the other two had little experience. (In the future, each physician will be expected to make a diagnosis on a cathode-ray tube (CRT) monitor.) Clinical information regarding the trauma was given to each reader, who had received training on the use of the display system. Viewing time was recorded for each film.
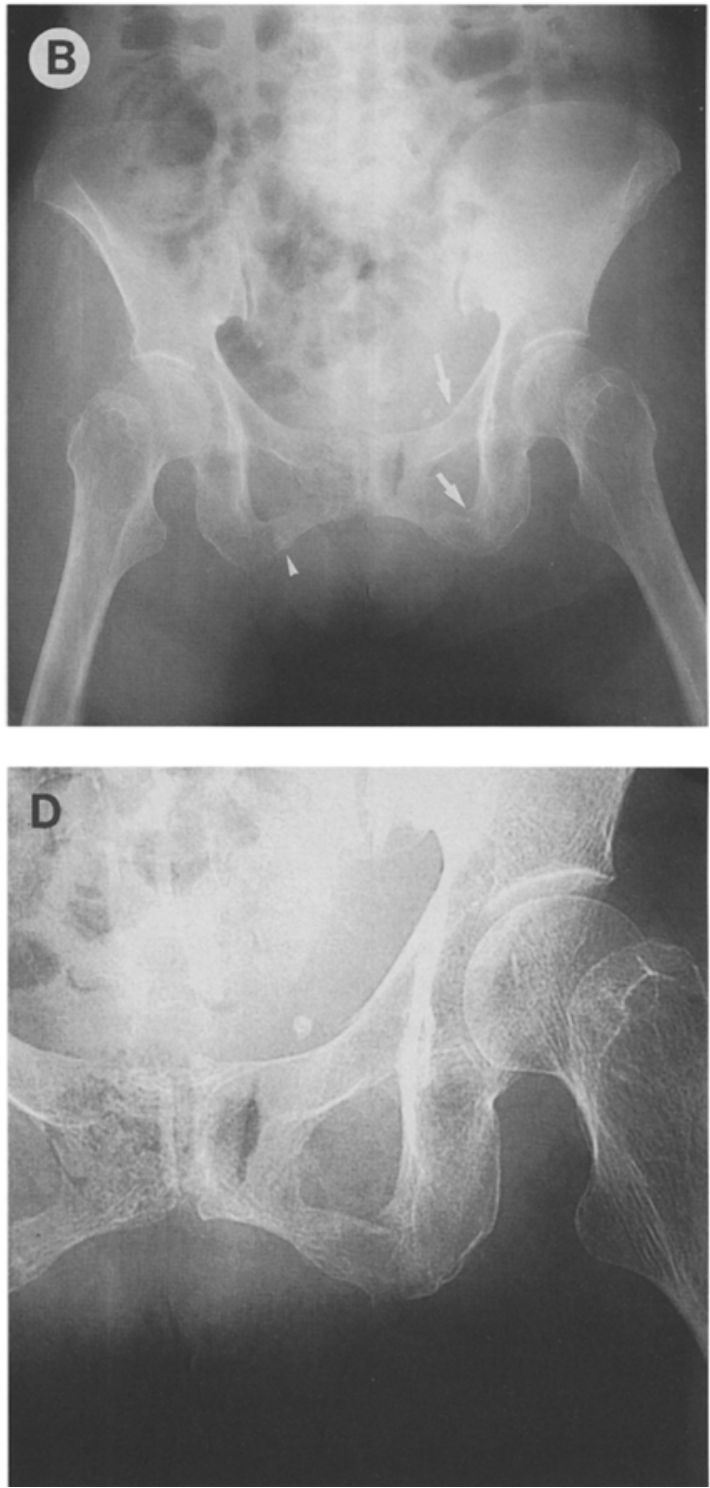

Fig 2. Left pubic and ischial fracture and misdiagnosis of right ischial fracture. On the hard-copy image processed with nonlinear conversion, it was difficult to detect the left pubic and ischial fractures (A). On the image with linear and edge enhancement, this fracture was easy to detect. All readers mistook a region of the right ischium (arrow) for a fracture on the image processed by linear conversion only (B). The bone parameter CRT image showed the fracture clearly (C). The magnification provided at the workstation makes it easier to see fractures (D). 


\section{Confidential Level}

A confidence level ( 1 through 4 ) was assigned to the interpretation: 1 , definitely normal; 2 , probably normal; 3 , probably abnormal; and 4 , definitely abnormal, indicating the highest level of confidence that the specified fracture was present.

\section{Statistical Analysis}

Receiver-operating characteristic (ROC) analyses were performed on all 54 fractures. The maximum likelihood method was used for curve-fitting interpolation.

\section{RESULTS}

\section{ROC Curve}

The radiologists with limited experience tended to overread the images. A tendency for

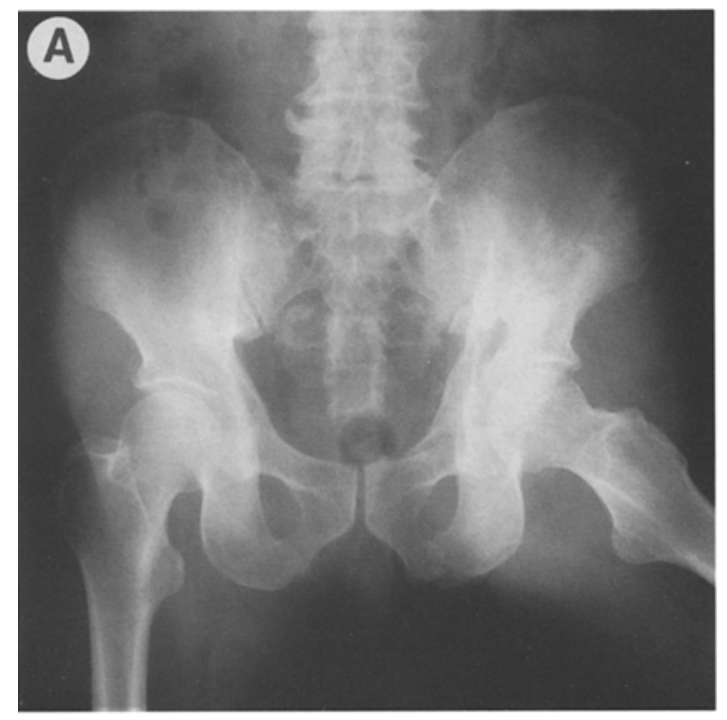

Fig 3. Fracture of the left ilium. It was hard to detect the left ilial fracture on the nonlinear-type image, which resembles a conventional radiograph (A). A linear-type image showed discontinuity of the bony cortex (B). A magnified image at the work station showed the fracture more clearly (C). interpretations to differ was seen between workstation- and linear-type images for reader no. 1 and between linear- and nonlinear-type images for reader no. 2 because of a large standard error. However, for skeletal fractures overall, there was no significant difference between linear- and nonlinear-type images and between the linear-type images and the workstation (Table 1). The linear-type image and magnification provided by the workstation were more useful for detecting small fractures than the nonlinear-type image (Figs 2, 3, and 4). Although the difference was not significant, the readers on the whole tended to overread the images at the workstation.
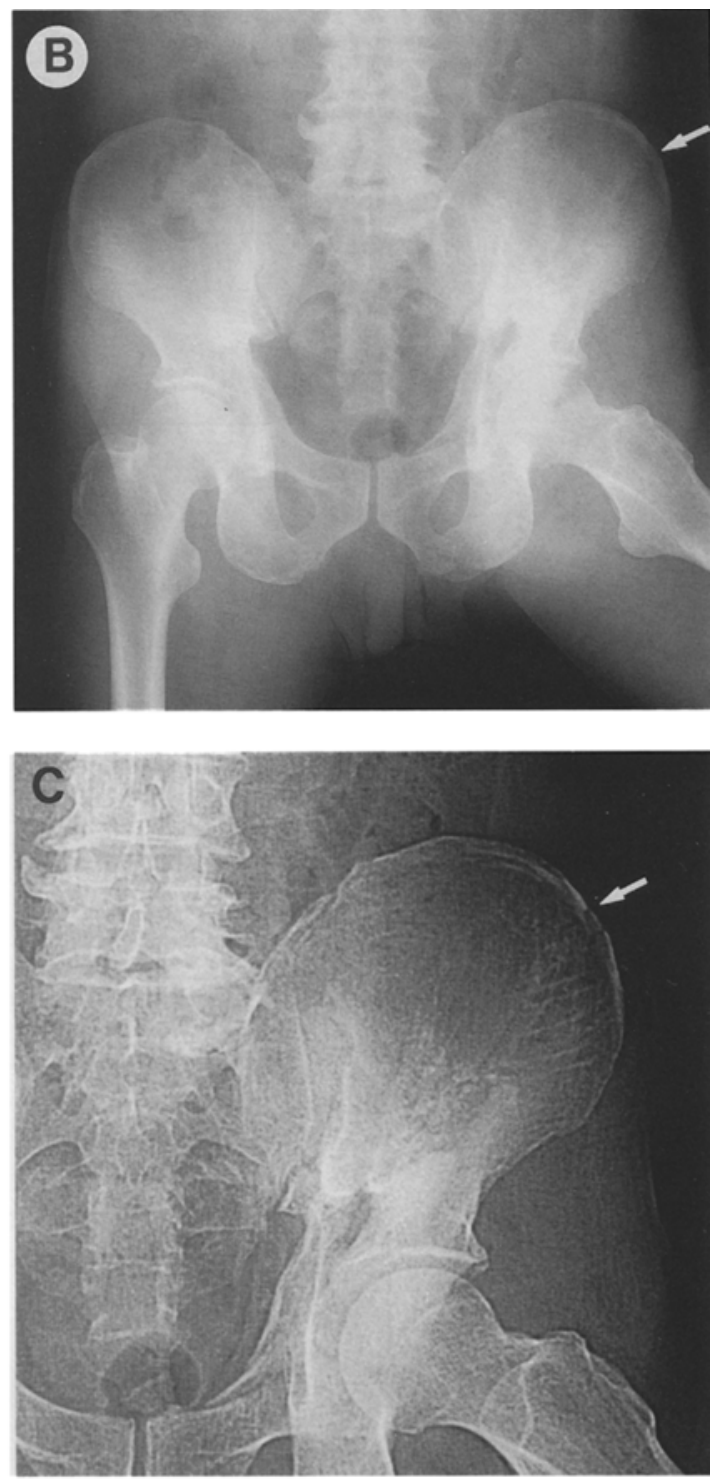

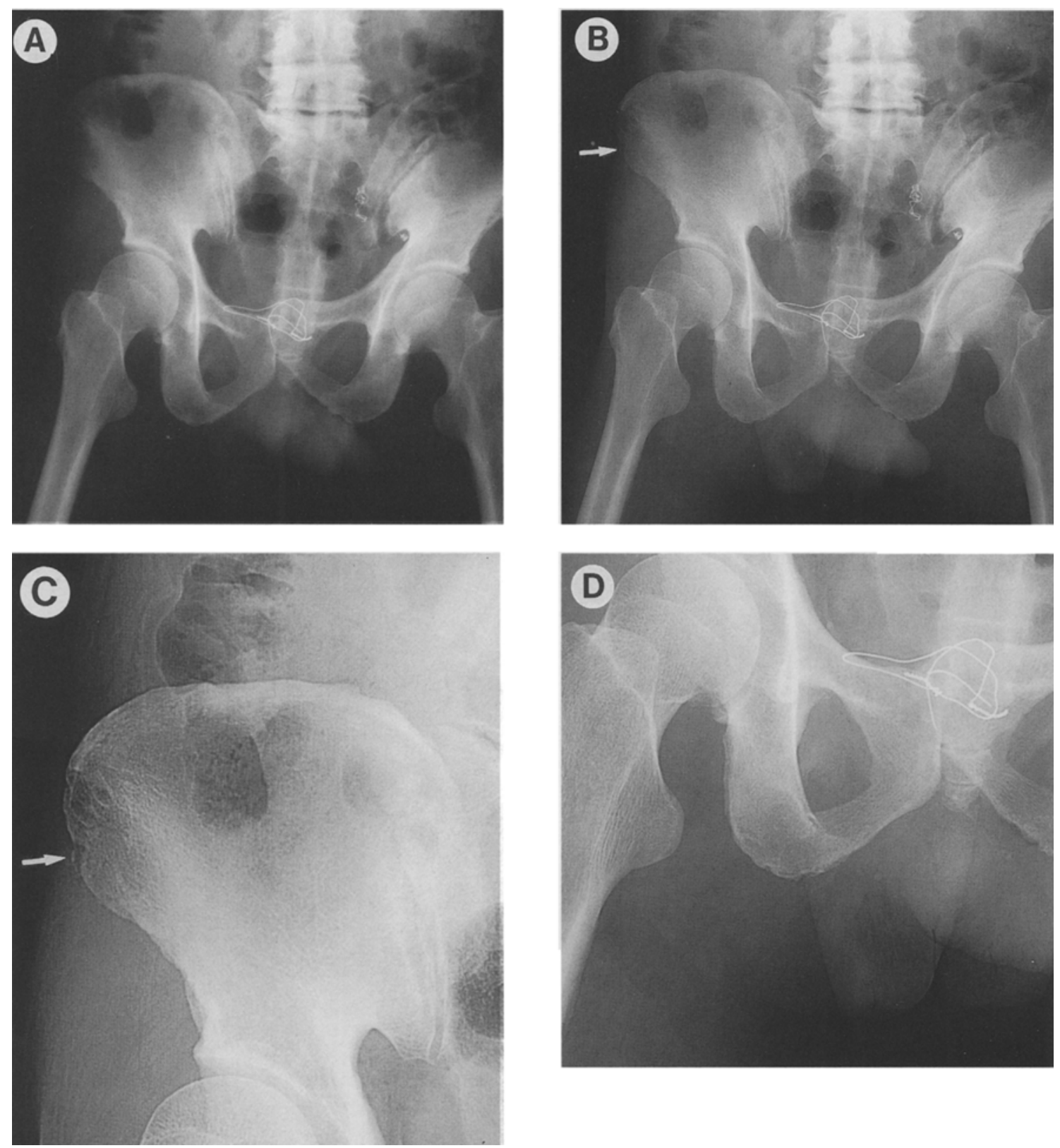

Fig 4. Fracture of the right ilium. Right ilial fracture could not be identified on the nonlinear-type image (A). The linear-type image showed small fragmentation near the ilium. A vague mask overshoot artifact that was not sharp (the so-called halo effect) could be seen adjacent to the right ischial cortical bone, and was mistaken for a fracture by two of the readers (B). Magnification was very useful for detecting small fractures (C).

\section{Reading Times}

The reading time of reader no. 3 was unreliable because a young physician tended to recall previous images. On the average, it took approximately 1.15 and 0.75 minutes for the older radiologists to read one nonlinear- and one linear-type image, respectively; reading a nonlinear-type image required approximately $35 \%$ longer than reading a linear-type image. It took 3 to 4 times longer to read an image on a workstation than a linear-type image (Fig 5).

\section{DISCUSSION}

Optimal Image Processing of Skeletal Fractures

Although there is no significant difference between a nonlinear-type image, which approxi- 

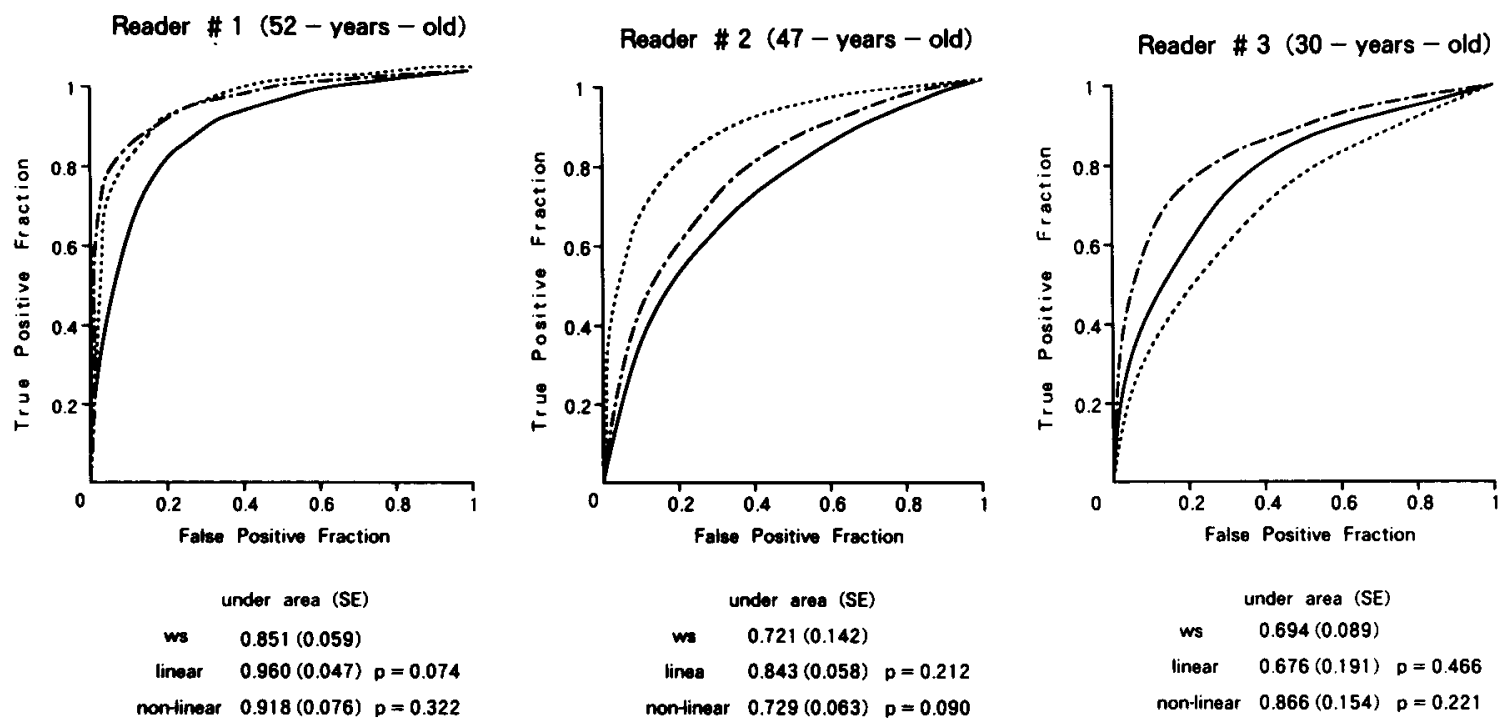

Fig 5. ROC curves for the three readers comparing the hard and soft copies processed with linear and nonlinear conversion. (-, workstation; $(\cdots)$, linear conversion; $(--)$, nonlinear conversion.

mates a conventional radiograph, and the lineartype image, it was easier to detect small fractures with the latter. Furthermore, magnification provided at workstation was useful.

A high-resolution display may be needed to find nondisplayed or subtle fractures, 4,5 The same radiation dose as that used in conventional film screening was necessary (so as not to decrease the image quality) because a significant difference in diagnostic accuracy with computed radiography images at 50\% exposure compared with conventional radiographs has been reported. ${ }^{2}$ The radiation dose should be reduced in accordance with the patient's age, gender, and clinical setting. For example, a follow-up study does not require a high image quality.

\section{Advantage of CRT Diagnosis}

The many functions available in a workstation system were advantageous in some respects. However, this availability increases the overall interpretation time and makes systems harder to learn and use. Prior or easy setting of parameters and functions is recommended to reduce reading time. (The merit in the use of a touch-panel keyboard has already been noted.) The most important advantages of CRT diagnosis may be that images can be stored and transmitted and one has easy access to recorded (ie, filed) images.

\section{Limitations of CRT Diagnosis}

The limitations of CRT diagnosis include short dynamic range, prolonged reading time caused by the limited number of combinations of windows and level settings as well as the availability of many other functions. However, the short dynamic range can be acceptable for skeletal fractures by adjusting the windows and level settings. The unlimited number of combinations of referred to above can be resolved by devising a preset menu for skeletal fractures.

\section{CONCLUSION}

There was no statistical difference between hard copies processed with linear and nonlinear conversion vis-a-vis diagnosis of skeletal fractures. However, it may be difficult to detect small fractures on images processed by nonlinear conversion.

For skeletal fractures, there was no significant difference between CRT diagnosis and that on hard copy processed with linear conversion and frequency enhancement.

Although overreading may increase, it is believed that CRT diagnosis is acceptable for skeletal fractures.

Linear conversion images are recommended as initial images for CRT diagnosis of skeletal fractures. Using a touch-panel keyboard makes the task of entering the bone parameters much easier. 


\section{REFERENCES}

1. Schaefer CM, Prokop M: Storage phosphor radiography of the chest. Radiology 186:314-315, 1993

2. Murphy MD, Quale JL, Martine NL, et al: Computed radiography in musculoskeletal imaging: State of the art. AJR 158:19-27, 1992

3. Prokop M, Galanski M, Oestmann JW, et al: Storage phosphor versus screen-film radiography: Effect of varying exposure parameters and unsharp mask filtering on the detectability of cortical bone defect. Radiology 177:109-113, 1990

4. Murphy MD, Bramble JM, Cook LT, et al: Nondisplaced fractures: Spatial resolution requirements for detection with skeletal imaging. Radiology 174:865-870, 1990

5. Wegryn SA, Piraino DA, Richmond BI, et al: Comparison of digital and conventional muskuloskeletal radiography: An observer performance study. Radiology 175:225228,1990 